\title{
Cerebral Sinus Venous Thrombosis in Indian Pediatric Patients with Acute Lymphoblastic Leukemia: Incidence, Risk Factors, and Outcome
}

\begin{abstract}
Background: Cerebral sinus venous Thrombosis (CSVT) is a rare complication of acute lymphoblastic leukemia (ALL) treatment, with an incidence rate ranging from $1.1 \%$ to $2.9 \%$. Steroids and L-asparaginase (LASP) are the major risk factors. Methods: The aim of this study was to find the incidence rate and risk factors for CSVT in our patients. Ninety-two pediatric ALL diagnosed and treated with ALL-Berlin-Frankfurt-Munster-95 protocol over a period of 5 years (2013-2017) were the subject of the study. Six (6.5\%) patients developed CSVT during their therapy and were further analyzed. Results: Six (five males and one female) patients had a CSVT event. The mean age at presentation was $13.5 \pm 4.1$ years. CSVT event occurred during the induction/re-induction phase of chemotherapy. The superior sagittal sinus was the most frequent site of thrombosis. The timing of CSVT was related to using of LASP and steroids. High-risk disease was associated with an increased risk of thrombosis. Infection and dehydration were other important risk factors. No association was observed between the risk of CSVT and age, sex, immunophenotype, and white blood cell count. All the patients were treated with low-molecular-weight heparin and one patient developed heparin-induced thrombocytopenia. No death occurred due to CSVT. Conclusions: The Incidence of CSVT was $6.5 \%$ in our study which is significantly high but comparable with studies from other Asian countries. It is, therefore, important to be vigilant for the detection of CSVT during treatment of ALL and initiate the appropriate treatment to prevent adverse events.
\end{abstract}

Keywords: Acute lymphoblastic leukemia, cerebral sinus venous thrombosis, L-asparaginase

\section{Introduction}

Acute lymphoblastic leukemia (ALL) is the most common childhood cancer, with an overall 5-year overall survival (OS) rate of $80 \%-86 \%$ and the 5 -year event-free survival (EFS) rate of approximately $78 \%-83 \%{ }^{[1]}$ Cerebral sinus venous thrombosis (CSVT) is an important complication of ALL therapy, with a reported prevalence rate of $1.1 \%-2.9 \%{ }^{[2-4]}$ It is associated with a mortality rate of $8 \%-13 \%$ and long-term morbidity. ${ }^{[3]}$ In a recent meta-analysis of several studies on venous thromboembolism (VTE) in pediatric ALL patients, the global risk of VTE has been estimated to be 5.2\%, and more than $50 \%$ of these events occurred in the central nervous system. ${ }^{[5]}$ Several risk factors have been identified for the occurrence of CSVT in ALL patients such as high-risk (HR) disease, T-cell immunophenotype, drugs such as L-asparaginase (LASP) and steroids,

This is an open access journal, and articles are distributed under the terms of the Creative Commons Attribution-NonCommercial-ShareAlike 4.0 License, which allows others to remix, tweak, and build upon the work non-commercially, as long as appropriate credit is given and the new creations are licensed under the identical terms.

For reprints contact: WKHLRPMedknow_reprints@wolterskluwer.com and presence of underlying inherited thrombophilia. ${ }^{[2,5-8]}$

Almost 10,000 new cases of childhood ALL are likely diagnosed in India each year. Even with an estimated risk of $5 \%$, over 500 new cases of thrombotic complications should be reported each year. ${ }^{[9]}$ However, there is a marked paucity of data on thrombotic complications in ALL patients from India and other Asian countries, with only a few published reports. ${ }^{[10-13]}$ The aim of this study was to find the incidence of CSVT and identify the risk factors in our pediatric ALL patients.

\section{Materials and Methods}

\section{Study population}

In this retrospective analysis, children with ALL between 1 and 18 years of age diagnosed and treated at our institution from January 2013 to December 2017 were the subject of the study. The clinical and laboratory data were retrieved from their medical case files.

\footnotetext{
How to cite this article: Garg A, Kashyap R, Lal H, Mishra P. Cerebral sinus venous thrombosis in Indian pediatric patients with acute lymphoblastic leukemia: Incidence, risk factors, and outcome. Indian J Med Paediatr Oncol 2020;41:523-9.
}

\section{Akanksha Garg ${ }^{1}$, Rajesh Kashyap', Hira Lal' ${ }^{2}$, Prabhakar Mishra ${ }^{3}$}

${ }^{1}$ Department of Hematology, Sanjay Gandhi Postgraduate Institute of Medical Sciences, Lucknow, Uttar Pradesh, India, ${ }^{2}$ Department of Radiology, Sanjay Gandhi Postgraduate Institute of Medical Sciences, Lucknow, Uttar Pradesh, India, ${ }^{3}$ Department of Biostatistics and Health Informatics, Sanjay Gandhi Postgraduate Institute of Medical Sciences, Lucknow, Uttar Pradesh, India

Submitted: 07-Oct-2019 Revised: 06-Feb-2020 Accepted: 10-Feb-2020 Published: 29-Aug-2020

Address for correspondence: Dr. Rajesh Kashyap, Department of Hematology, Sanjay Gandhi Postgraduate Institute of Medical Sciences, Rae Bariely Road, Lucknow - 226014 , Uttar Pradesh, India. E-mail: rajkashyapmd@yahoo. co.in

Access this article online Website: www.ijmpo.org DOI: 10.4103/ijmpo.ijmpo_206_19 Quick Response Code:

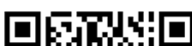

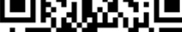
27.5.7. 1: $4,7,7$ 回布院 
All the children who developed CSVT were further analyzed. The diagnosis and risk classification was according to the ALL-Berlin-Frankfurt-Munster (BFM)-95 protocol. $^{[14]}$ The patients were risk-stratified according to age, initial white blood cell (WBC) count, day 8 response to prednisolone, T-immunophenotype, and molecular re-arrangement into standard risk, medium risk (MR), and HR groups. The treatment protocol at our institute is a modification of ALL-BFM-95 protocol. $^{[14]}$ The induction chemotherapy consisted of four-drug phase A (daily oral prednisolone $60 \mathrm{mg} / \mathrm{m}^{2}$ days $1-28$, once weekly intravenous vincristine $1.5 \mathrm{mg} / \mathrm{m}^{2}$ and daunorubicin $30 \mathrm{mg} / \mathrm{m}^{2} \times 4$ weeks and twice weekly intravenous LASP $5000 \mathrm{u} / \mathrm{m}^{2} \times 8$ doses) followed by phase B (intravenous cyclophosphamide $1000 \mathrm{mg} / \mathrm{m}^{2}$ on days 34 and 64, intravenous cytosine arabinoside $75 \mathrm{mg} /$ $\mathrm{m}^{2} 4$ blocks of 4 days each between weeks 6 and 9, and daily oral 6-mercaptopurine (6-MP) $\left.50 \mathrm{mg} / \mathrm{m}^{2}\right)$. The consolidation/extra-compartment phase consisted of a 24-h infusion of methotrexate (MTX) $5.0 \mathrm{~g} / \mathrm{m}^{2}$ every 2 weeks and cytosine arabinoside $200 \mathrm{mg} / \mathrm{m}^{2}$ for a total of 4 doses and daily oral 6-MP $25 \mathrm{mg} / \mathrm{m}^{2}$. The re-induction phase comprised phase A (weekly intravenous vincristine $1.5 \mathrm{mg} / \mathrm{m}^{2}$ and doxorubicin $30 \mathrm{mg} / \mathrm{m}^{2} \times 4$ weeks, LASP $10,000 \mu / \mathrm{m}^{2} \times 4$ doses during weeks $19-20$, and daily oral dexamethasone $10 \mathrm{mg} / \mathrm{m}^{2}$ ) followed by phase B (single dose of intravenous cyclophosphamide $1000 \mathrm{mg} / \mathrm{m}^{2}$ at week 22 and two 4 days block of intravenous cytosine arabinoside $75 \mathrm{mg} / \mathrm{m}^{2}$ at weeks 23 and 24 and oral 6-thioguanine (6-TG) $\left.60 \mathrm{mg} / \mathrm{m}^{2}\right)$. Escherichia coli LASP was used in our patients. Central nervous system (CNS)-directed therapy consisted of intrathecal MTX (IT-MTX) $12 \mathrm{mg} /$ dose administered in induction phase ( 2 and 3 doses in phase $\mathrm{A}$ and $\mathrm{B}$, respectively), 4 doses in extra-compartment phase (every two weeks), and 2 doses in re-induction phase B. Patients with HR disease received 2 additional doses of IT-MTX in induction phase A and cranial radiotherapy $18 \mathrm{~Gy}$ in 10 fractionated doses after re-induction $\mathrm{B}$ phase of the chemotherapy protocol. The maintenance phase consisted of daily oral 6-MP $50 \mathrm{mg} / \mathrm{m}^{2}$ and weekly oral MTX $20 \mathrm{mg} / \mathrm{m}^{2}$. The duration of treatment in females and males was 104 and 156 weeks from the start of therapy, respectively.

\section{Documentation of thrombotic events}

The diagnosis of CSVT was done by standard radiological techniques as per the institute guidelines. Thrombosis of the cerebral vessels was diagnosed by computed tomography scan, magnetic resonance imaging (MRI) and magnetic resonance venography (MRV). Compression ultrasonography (USG) and color Doppler USG were performed to rule out deep vein thrombosis of extremities. Repeat radiological evaluation was done post 3 months of anticoagulation therapy to look for resolution of thrombosis.

\section{Evaluation of hemostatic defects}

All patients with thrombosis underwent laboratory screening for inherited thrombophilia. Laboratory evaluation included complete blood count, prothrombin time, activated partial thromboplastin time, fibrinogen level, and D-dimer assay. Prothrombotic state evaluation included measurement of antithrombin (AT) III and protein C activities by chromogenic assay and free protein $\mathrm{S}$ antigen levels by ELISA technique chromogenic assay. Factor V Leiden, prothrombin G20210A mutation, and MTHFR gene mutation analysis were done by molecular techniques.

\section{Management of thrombosis}

All the patients with symptomatic CSVT were treated with low-molecular-weight heparin (LMWH) (enoxaparin) $1 \mathrm{mg} / \mathrm{kg}$ every 12 hourly for the first 2 weeks and then once a day for a minimum of 3-month duration. At the end of 3 months, patients were re-evaluated radiologically for the resolution of thrombosis. If recanalization of the thrombosed vessel occurred then LMWH was stopped, if there was no or only partial recanalization of the thrombosed sinuses it was given for an additional period 3 months as per the recommended guidelines. ${ }^{[15]}$ During the treatment with $\mathrm{LMWH}$, patients were monitored for the occurrence of any adverse events such as bleeding and heparin-induced thrombocytopenia (HIT). Fondaparinux (indirect Factor $\mathrm{Xa}$ inhibitor) $0.1 \mathrm{mg} / \mathrm{kg} /$ day was used in patients who developed HIT.

\section{Statistical analysis}

Continuous variables were presented using mean \pm standard deviation while categorical variables were in frequency (\%). The unpaired $t$-test was used to compare the means while Fisher's exact test for proportions between CSVT and non-CSVT groups. Univariate binary logistic regression analysis was performed to compute odds ratio with a corresponding 95\% confidence interval. $P<0.05$ was considered as statistically significant. Statistical analysis was performed using the Statistical Package for the Social Sciences, version 23 (SPSS-23, IBM, Chicago, Illinois, USA).

In this retrospective study, there are no patient-identifying data, and no new tests or interventions were performed. The study was carried out as per the institute's IRB and ethics committee's rules and guidelines, and no approval was required.

\section{Results}

Over a period of 5 years (January 2013-December 2017), 238 patients were diagnosed with ALL and were treated at our center. Ninety-two (68 males and 24 females) patients were between the ages of 1 and 18 years. The mean age was $12.01 \pm 4.38$ (range: $4-18$ ) years. Seventy-four (80.4\%) patients had B-cell immunophenotype and the remaining $18(19.6 \%)$ had T-ALL. The majority of the patients had MR (76.1\%) and HR (14.1\%) diseases. The comparative baseline clinical and laboratory profile of pediatric ALL patients with and without CSVT is shown in Table 1. 


\begin{tabular}{|c|c|c|c|c|c|}
\hline \multirow[t]{2}{*}{ Parameter } & \multirow[t]{2}{*}{ CSVT group $(n=6)$} & \multirow[t]{2}{*}{ Non-CSVT group $(n=86)$} & \multirow[t]{2}{*}{$P$} & \multicolumn{2}{|c|}{ Univariate binary logistic regression analysis } \\
\hline & & & & OR & $95 \%$ CI \\
\hline \multicolumn{6}{|l|}{ Age (years) } \\
\hline Mean \pm SD (range) & $13.5 \pm 4.46(7-18)$ & $11.91 \pm 4.41(4-18)$ & 0.395 & 1.09 & $0.89-1.33$ \\
\hline \multicolumn{6}{|c|}{ Age groups (years), $n(\%)$} \\
\hline$\geq 10$ & $4(66.7)$ & $56(65.1)$ & 0.997 & 1.07 & $0.19-6.19$ \\
\hline$<10$ & $2(33.3)$ & $30(34.9)$ & & & \\
\hline \multicolumn{6}{|l|}{ Gender, $n(\%)$} \\
\hline Male & $5(83.3)$ & $63(73.3)$ & 0.999 & 1.83 & $0.20-16.47$ \\
\hline Female & $1(16.7)$ & $23(26.7)$ & & & \\
\hline \multicolumn{6}{|l|}{ ALL phenotype, $n(\%)$} \\
\hline B-cell & $4(66.7)$ & $70(81.4)$ & 0.334 & 0.46 & $0.08-2.72$ \\
\hline T-cell & $2(33.3)$ & $16(18.6)$ & & & \\
\hline \multicolumn{6}{|l|}{ Risk stratification, $n(\%)$} \\
\hline High risk & $4(66.7)$ & $9(10.5)$ & 0.006 & $17.11^{\#}$ & $2.74-106.93$ \\
\hline Medium risk & $2(33.3)$ & $68(79.0)$ & & & \\
\hline Standard risk & 0 & $9(10.5)$ & & & \\
\hline \multicolumn{6}{|l|}{$\begin{array}{l}\text { White blood cell count } \\
\text { (at diagnosis) }\end{array}$} \\
\hline Mean \pm SD & $70.2 \pm 97.11$ & $39.41 \pm 71.50$ & 0.329 & 1.004 & 0.996-1.012 \\
\hline Median $\times 10^{9} / \mathrm{L}$ & 35.2 & 9.85 & & & \\
\hline CNS disease & 0 & $7(8.1)$ & - & - & - \\
\hline Prothrombotic defects & none & None & - & - & - \\
\hline
\end{tabular}

\section{Central nervous system thrombosis}

Six patients $(6.5 \%)$ developed CNS thrombosis during ALL therapy, and all of them had CSVT [Table 1]. The diagnosis of CSVT was based on clinical symptoms and signs and was confirmed by magnetic resonance imaging (with or without contrast) and MR venography of the brain [Figure 1a and $\mathrm{b}$ ]. The mean age at presentation was $13.5 \pm 4.1$ (range: 7-18; median: 15) years. Four patients $(66.7 \%)$ had B-ALL and the remaining $2(33.3 \%)$ had T-ALL. All the six patients had MR/HR disease. The mean WBC count at diagnosis in these patients was $70.21 \pm 97.11$ (range: $1.93-281.0$; median: $35.2 \times 10^{9} / \mathrm{L}$ )

The most common sites of CSVT included the sagittal sinus (5 cases), transverse sinus (4 cases), and sigmoid sinus ( 2 cases). Seizures (focal or generalized) were the presenting feature in all the six cases with CSVT. Headache was present in $83.3 \%$ of cases. Neurological deficit was seen in only 1 patient (patient no. 3) who presented with left-sided hemiparesis and facial nerve palsy. This patient had extensive sinus thrombosis involving the superior sagittal sinus, bilateral transverse sinuses, straight sinuses, and cortical veins [Table 2].

Timing and risk factors

Five $(83.3 \%)$ of the six patients with CSVT developed thrombosis during induction chemotherapy and one

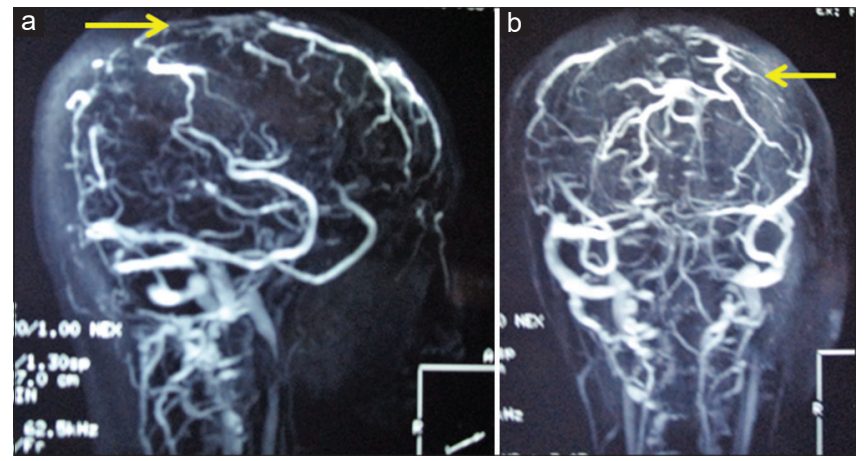

Figure 1: (a and b) Medium-risk venography (sagittal and coronal views) showing a partial filling defect of superior sagittal sinus suggestive of thrombosis (indicated by arrow)

patient (patient no. 4) during the re-induction A phase. In two patients, the CSVT occurred in the $1^{\text {st }}$ week of induction chemotherapy while receiving oral prednisolone alone. In the remaining four patients, the CSVT event occurrence correlated with the use of LASP and steroids [Table 2]. In two patients (cases 3 and 6), bacterial sepsis was the additional risk factor for CSVT. None of our six patients had an inherited prothrombotic state.

On statistical analysis, no significant association was observed between the occurrence of CSVT and age $>10$ years, gender, ALL immunophenotype, and WBC count in our study. The proportion of CSVT was 


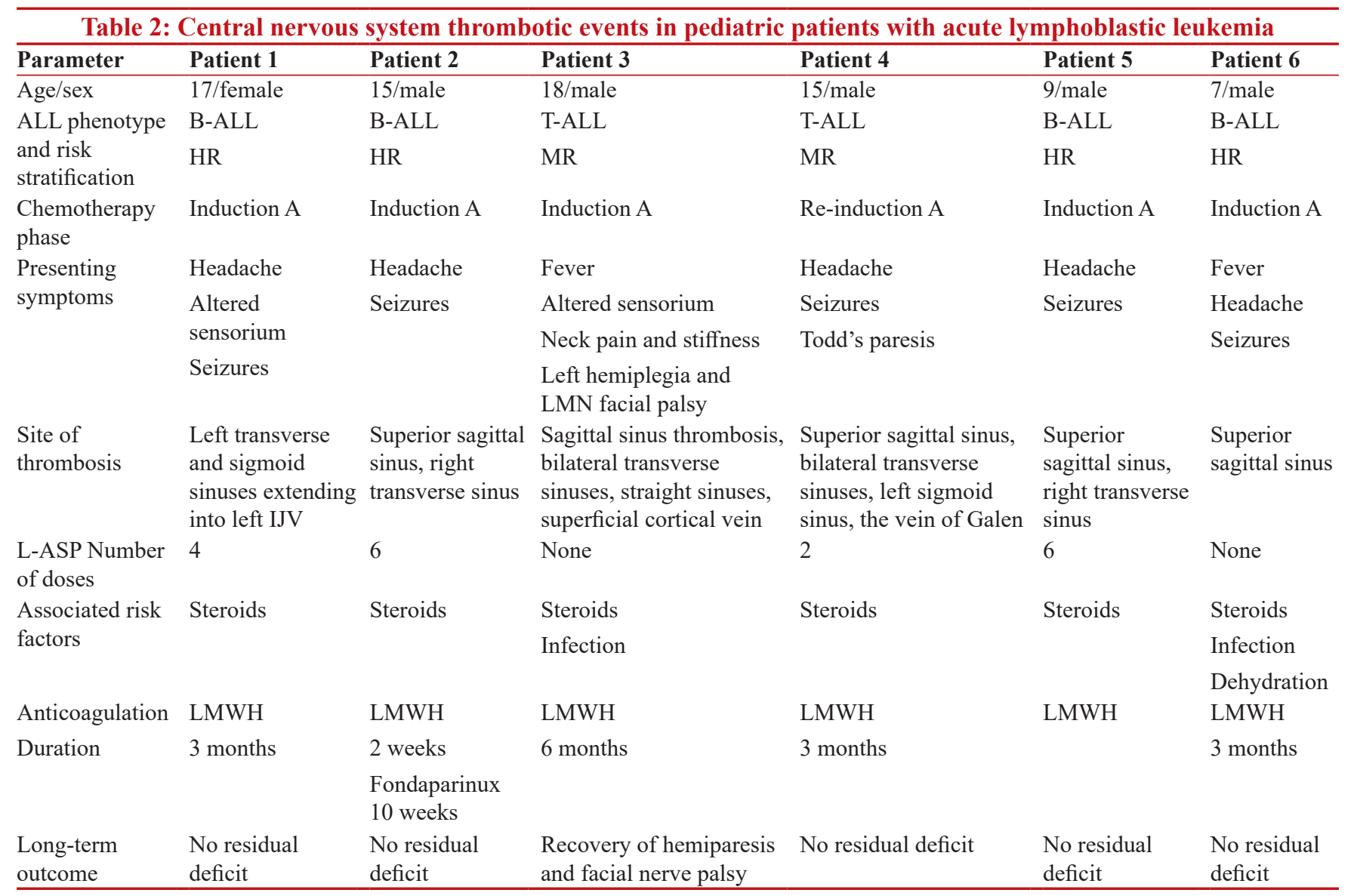

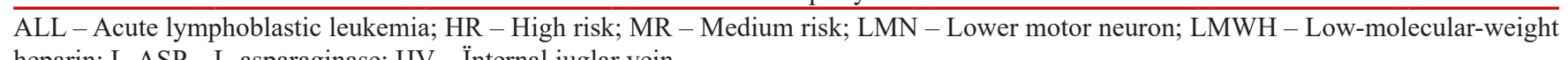
heparin; L-ASP - L-asparaginase; IJV - Ïnternal juglar vein

significantly different between the three groups of risk grading $(P=0.006)$. High-risk groups had 17.11 times higher risk of having CSVT as compared to rest patients which were found as statistically significant $(P<0.05)$ [Table 1].

\section{Treatment and long-term outcome}

All the six patients were initially treated with LMWH (enoxaparin). Patient no. 2 developed thrombocytopenia after 2 weeks of anticoagulation with LMWH. ELISA test for heparin-PF4 antibodies was positive suggestive of HIT. Fondaparinux was then used in place of LMWH. Treatment was given for 3 months, and once complete recanalization was documented, the anticoagulation therapy was stopped. Patient no. 3 showed partial recanalization at the end of 3 months of therapy, and anticoagulation was continued for further 3 months.

Chemotherapy was continued as per the protocol once the patient was neurologically stable. LASP was omitted in the phase of chemotherapy during which the thrombotic event occurred. Re-exposure to LASP was done in the subsequent phases of treatment as per the protocol. None of the patients had a fresh thrombotic event when re-exposed to LASP in the subsequent phases of chemotherapy protocol. Pegylated LASP was used in patient no. 6, and no adverse events were documented.
IT-MTX was withheld for the first $72 \mathrm{~h}$ after the occurrence of CSVT event and initiation of anticoagulation therapy to reduce the possible risk of bleeding and any neurological adverse event. It was then given as per the protocol. LMWH was stopped $24 \mathrm{~h}$ before scheduled lumbar puncture and restarted $12 \mathrm{~h}$ after the procedure. All the six patients received all the doses of IT-MTX as per the protocol.

No mortality occurred in our patients with CSVT, and patient no. 3 who had hemiplegia at presentation showed improvement in his neurological status. Patient no. 5 had disease relapse during the maintenance phase of chemotherapy [Table 2].

\section{Discussion}

CSVT is a well-known complication of ALL chemotherapy, and its prevalence ranges from $1.1 \%$ to $2.9 \%{ }^{[2-4]}$ It is associated with a mortality rate of $8 \%-10 \%$ and a significant neurological deficit. ${ }^{[3,4]}$ Caruso et al. in a meta-analysis of 17 studies in the pediatric population observed a global risk of $5.2 \%$ for VTE and $31 \%$ of äll the thrombotic events in them involved the cerebral venous sinuses with a risk of $2.9 \% .^{[5]}$ In contrast, in the PARKKA study which included radiologically documented 
asymptomatic VTE events, the majority of the events involved upper venous system (36.7\%) and only $4.5 \%$ of the VTE events were CSVT with an overall incidence of $1.7 \%{ }^{[16]}$ Similarly, Athale et al. reported the incidence of CSVT to be $2.2 \%$ in their study and it accounted for $20 \%$ of all VTE events. ${ }^{[17]}$

There are very few studies on CSVT in pediatric ALL patients from India and other Asian countries, and its reported incidence ranged between $1.5 \%$ and $6.2 \% \cdot{ }^{[10-13]}$ Malhotra et al. diagnosed 7 cases of CSVT among 467 (1.5\%) pediatric ALL patients treated at their center over a 17-year period. Six of the CSVT events occurred during the induction phase and 1 during the re-induction phase. ${ }^{[10]}$ Lee et al. from South Korea in their study of VTE following LASP treatment for lymphoid malignancy in both pediatric and adult patients found that the overall incidence of VTE was $3.5 \%$ and CSVT constituted $4.7 \%$ of these events in their pediatric population with ALL. ${ }^{[1]}$ In another study from Lebanon, Ghanem et al. have reported a higher incidence of CSVT. Thirteen of their 209 patients treated with modified St. Jude total XV treatment protocol for ALL developed CSVT, with an incidence rate of $6.2 \% \cdot{ }^{[12]}$ Torun et al. have reported the CSVT incidence to be $2.4 \%$ in their 82 ALL patients treated with BFM-90/95 protocol. ${ }^{[13]}$ In our study, the incidence rate of CSVT was $6.5 \%$, and it is higher when compared to the studies from European countries and the USA. ${ }^{[2,3,16,17]}$ However, it is comparable to findings from other Asian countries. ${ }^{[1,12]}$ These variations in incidence rates are multifactorial; it is influenced by patient characteristics, the intensity of the treatment protocol used, and preexistent prothrombotic factors. ${ }^{[2,4,11-13]}$

The CSVT events have been documented to occur during treatment of ALL, and it is rare at diagnosis or during the maintenance phase of therapy. ${ }^{[2,5]}$ In three-fourth of the cases, the CSVT has occurred during the induction phase of therapy and correlated with the use of LASP and steroids during this phase of therapy..$^{[3-5,11,13]}$ The association between CSVT and the use of LASP has been documented in patients treated with a Dana Farber Cancer Institute ALL protocol. In this protocol, the thrombotic event occurred during the intensification phase during which the patients received weekly LASP and pulses of steroids. ${ }^{[17]}$ It has been observed that CSVT usually occurred within 2-3 weeks of LASP administration. ${ }^{[3]}$ Sutor et al. in a retrospective study found that in ALL patients treated with BFM-90 protocol, $80 \%$ of the symptomatic events occurred during the induction phase of therapy when they were receiving steroids and LASP. ${ }^{[18]}$

It has been observed that the risk of thrombosis is low when steroids and LASP are used separately, but the risk increases 10 folds when both the drugs are used together. ${ }^{[19,20]}$ Studies comparing the incidence of symptomatic thrombotic events in ALL patients treated with BFM protocol versus COALL regimen have observed the risk of thrombosis to be $11 \%$ in the former group, and it was only $1.5 \%-2 \%$ in the latter group. This difference was statistically significant. ${ }^{[19,20]}$ LASP reduces AT III levels, and steroids have shown to raise Factor VIII and von Willebrand factor levels. These associated hemostatic changes predispose to thrombotic risk. ${ }^{[2]}$ LASP is an important component of ALL therapy. Inadequate LASP dosing in ALL patients with VTE due to drug interruption or dose reduction has been shown to be associated with lower EFS. ${ }^{[22,23]}$ Grace et al. have observed that patients with VTE who receive at least $85 \%$ of the planned LASP dosage after rechallenge have a similar EFS and OS compared to patients without VTE. ${ }^{[24]}$ There are conflicting results regarding the risk of recurrent VTE on re-exposure to LASP. Few studies have shown that re-exposure to LASP is safe and the risk of another thrombotic event is minimal. ${ }^{[2,4,12]}$ In contrast, Klaassen et al. have shown that there was an increased rate of recurrent VTE in their patients when challenged with LASP. ${ }^{[25]}$ Pegylated LASP appears to have a similar risk of VTE when compared to E. coli LASP. $^{[2]}$

Additional risk factors for CSVT include T-cell immunophenotype, high-risk disease, infections, and dehydration. ${ }^{[3,4,12]}$ The role of inherited prothrombotic defects and the risk of VTE in pediatric ALL patients is debatable. A meta-analysis of five studies by Caruso et al. has shown that the presence of at least one thrombophilia state is associated with an 8-fold increase in the risk of VTE in these patients. ${ }^{[5]}$ A German multicenter study found that the presence of protein $\mathrm{C}, \mathrm{S}$, and AT III defect was associated with a maximum risk of VTE ${ }^{[26]}$ In contrast, the PARKAA study failed to find any association between the risk of VTE and prothrombotic defects. ${ }^{[16]}$

The short-term impact of CSVT in these patients is interruptions and prolongation of ALL treatment. ${ }^{[3-4,12,18]}$ The data on the overall negative impact of VTE on the long-term survival of ALL patients is limited. ${ }^{[12,22,23,27]}$ Ghanem et al. have reported that the occurrence of CSVT had no adverse impact on relapse rate or OS in their study. ${ }^{[12]}$ More recently, Klaassen et al. have observed from their study that there is no association between the occurrence of VTE and EFS in these patients. ${ }^{[28]}$ CSVT is associated with significant mortality rates of $8 \%-13 \%$ and long-term neurological sequelae and disability rates of $20 \%-30 \%{ }^{[3,28,29]}$

In our study, the incidence of CSVT was $6.5 \%$, and children with HR disease were at a greater risk of developing CSVT during ALL therapy. The presence of associated risk factors, bacterial sepsis, and dehydration can possibly explain the higher incidence of CSVT in our study. We also observed that CSVT was not associated with any negative impact on the OS of our ALL patients. No mortality was observed in our CSVT patients. Our study had certain limitations that it was a retrospective observational study conducted over 
a 5-year period only, and these two factors could have influenced the incidence rate of CSVT in our patients.

\section{Conclusion}

The risk of developing CSVT in children with ALL during therapy although small is significant. It occurs due to a combination of factors linked to the disease itself to its treatment, and children with high-risk disease are at a greater risk of developing CSVT. The clinicians should be vigilant, and early recognition of prodromal symptoms such as headache and seizures contributes to earlier detection of cerebral venous thrombosis and prompt initiation of anticoagulation therapy with improved outcome.

\section{Financial support and sponsorship}

Nil.

\section{Conflicts of interest}

There are no conflicts of interest.

\section{References}

1. Pui CH, Evans WE. Treatment of acute lymphoblastic leukemia. N Engl J Med 2006;354:166-78.

2. Qureshi A, Mitchell C, Richards S, Vora A, Goulden N. Asparaginase-related venous thrombosis in UKALL 2003- re-exposure to asparaginase is feasible and safe. $\mathrm{Br} \mathrm{J}$ Haematol 2010;149:410-3.

3. Ranta S, Tuckuviene R, Makipernaa A, Albertsen BK, Frisk T, Tedgard $\mathrm{U}$, et al. Cerebral venous thrombosis in children with acute lymphoblastic leukemia - A multicentric study from the Nordic society of pediatric hematology and oncology. $\mathrm{Br} \mathrm{J}$ Hematol 2015; 168:547-52.

4. Musgrave KM, van Delft FW, Avery P, Clack RM, Chalmers EA, Qureshi A, et al. Cerebral sinovenous thrombosis in children and young adults with acute lymphoblastic leukemia - A cohort study from the United Kingdom. Br J Hematol 2017;179:667-8.

5. Caruso V, Iacoviello L, Di Castelnuovo A, Storti S, Mariani G, de Gaetano G, et al. Thrombotic complications in childhood acute lymphoblastic leukemia: A meta-analysis of 17 prospective studies comprising 1752 pediatric patients. Blood 2006;108:2216-22.

6. Payne JH, Vora AJ. Thrombosis and acute lymphoblastic leukaemia. Br J Haematol 2007;138:430-45.

7. Nowak-Göttl U, Kenet G, Mitchell LG. Thrombosis in childhood acute lymphoblastic leukaemia: Epidemiology, aetiology, diagnosis, prevention and treatment. Best Pract Res Clin Haematol 2009;22:103-14.

8. Athale UH, Chan AK. Thrombosis in children with acute lymphoblastic leukemia: Part I. Epidemiology of thrombosis in children with acute lymphoblastic leukemia. Thromb Res 2003;111:125-31.

9. Kulkarni KP, Arora RS, Marwaha RK. Survival outcome of childhood acute lymphoblastic leukemia in India: A resource-limited perspective of more than 40 years. J Pediatr Hematol Oncol 2011;33:475-9.

10. Malhotra P, Jain S, Kapoor G. Symptomatic cerebral sinovenous thrombosis associated with L-asparaginase in children with acute lymphoblastic leukemia: A single institution experience over 17 years. J Pediatr Hematol Oncol 2018;40:e450-3.

11. Lee JH, Lee J, Yhim HY, Oh D, Bang SM. Venous thromboembolism following L-asparaginase treatment for lymphoid malignancies in Korea. J Thromb Haemost 2017;15:655-61.

12. Ghanem KM, Dhayni RM, Al-Aridi C, Tarek N, Tamim H, Chan AK, et al. Cerebral sinus venous thrombosis during childhood acute lymphoblastic leukemia therapy: Risk factors and management. Pediatr Blood Cancer 2017;64:e266-94.

13. Torun YA, Patiroglu T, Ozdemir MA, Ozkul Y, Ekici A, Karakukcu M. Inherited prothrombotic risk factors in Turkish children with acute lymphoblastic leukemia: Significance of concomitant genetic mutation. Clin Appl Thromb Hemost 2012;18:218-21.

14. Möricke A, Reiter A, Zimmermann M, Gadner H, Stanulla M, Dördelmann $\mathrm{M}$, et al. Risk-adjusted therapy of acute lymphoblastic leukemia can decrease treatment burden and improve survival: Treatment results of 2169 unselected pediatric and adolescent patients enrolled in the trial ALL-BFM 95. Blood 2008;111:4477-89.

15. Zia AN, Chitlur M. Management of thrombotic complications in acute lymphoblastic leukemia. Indian J Pediatr 2013;80:853-62.

16. Mitchell LG, Andrew M, Hanna K, Abshire T, Halton J, Anderson $\mathrm{R}$, et al. A prospective study determining the prevalence of thrombotic events in children with acute lymphoblastic leukemia and a central venous line who are treated with L-asparaguses: Results of the prophylactic anti-thrombin replacement in kids with acute lymphoblastic leukemia treated with asparaginase (PARKAA) study. Cancer 2003;97:508-16.

17. Athale UH, Siciliano SA, Crowther M, Barr RH, Chan AK. Thromboembolism in children with acute lymphoblastic leukemia treated on Dana Farber Cancer Institute protocols: Effect on age and risk stratification of disease. Br J Hematol 2005;129:803-10.

18. Sutor AH, Mall V, Thomas KB. Bleeding and thrombosis in children with acute lymphoblastic leukaemia, treated according to the ALL-BFM-90 protocol. Klin Padiatr 1999;211:201-4.

19. Mauz-Körholz C, Nürnberger W, Irsfeld H, Körholz D, Göbel U. Low rate of severe venous thromboses in children with ALL treatment according to COALL-92 and -97 protocol. Klin Padiatr 1999;211:215-7.

20. Nowak-Göttl U, Heinecke A, von Kries R, Nürnberger W, Münchow N, Junker R. Thrombotic events revisited in children with acute lymphoblastic leukemia: Impact of concomitant Escherichia coli asparaginase/prednisone administration. Thromb Res 2001;103:165-72.

21. Athale UH, Chan AK. Thrombosis in children with acute lymphoblastic leukaemia: Epidemiology of thrombosis in children with acute lymphoblastic leukaemia. Part II. Pathogenesis of thrombosis in children with acute lymphoblastic leukemia. Effects of the disease and therapy. Thrombosis Res 2003;111:199-212.

22. Hunault-Berger $M$, Chevallier $P$, Delain $M$, Bulabois $C E$, Bologna S, Bernard $\mathrm{M}$, et al. Changes in antithrombin and fibrinogen levels during induction chemotherapy with L-asparaginase in adult patients with acute lymphoblastic leukemia or lymphoblastic lymphoma. Use of supportive coagulation therapy and clinical outcome: The CAPELAL study. Haematologica 2008;93:1488-94.

23. Ku GH, White RH, Chew HK, Harvey DJ, Zhou H, Wun T. Venous thromboembolism in patients with acute leukemia: Incidence, risk factors, and effect on survival. Blood 2009;113:3911-7.

24. Grace RF, Dahlberg SE, Neuberg D, Sallan SE, Connors JM, Neufeld EJ, et al. The frequency and management of asparaginase-related thrombosis in paediatric and adult patients with acute lymphoblastic leukaemia treated on Dana-Farber 
Cancer Institute consortium protocols. $\mathrm{Br} \quad \mathrm{J}$ Haematol 2011;152:452-9.

25. Klaassen IL, van Els AL, van de Wetering MD, van Ommen $\mathrm{CH}$. Increasing incidence and recurrence rate of venous thromboembolism in paediatric oncology patients in one single centre over 25 years. Thromb Haemost 2017;117:2156-62.

26. Nowak-Göttl U, Wermes C, Junker R, Koch HG, Schobess R, Fleischhack G, et al. Prospective evaluation of the thrombotic risk in children with acute lymphoblastic leukemia carrying the MTHFR TT 677 genotype, the prothrombin G20210A variant, and further prothrombotic risk factors. Blood 1999;93:1595-9.

27. Ogawa C, Ohara A, Manabte A, Hanada R, Takashi H,
Koh K, et al. Treatment outcome of discontinued L-asparaginase in children with standard-risk acute lymphoblastic leukemia: Tokyo children cancer study group (TCCSG) study L99-15 (abstract). Blood 2005;106:258a.

28. Klaassen IL, Lauw MN, Flocco M, van der Sluis IM, Pieters R, Middeldorp S, et al. Venous thromboembolism in a large cohort of children with acute lymphoblastic leukemia: Risk factors and effect on prognosis. Res Pract Thromb Haemost. Res Pract Thromb Haemost 2019;3:234-41.

29. Eden D, Hipkins R, Bradbury CA. Cerebral thrombotic complications related to 1-asparaginase treatment for acute lymphoblastic leukemia: Retrospective review of 10 cases. Clin Appl Thromb Hemost 2016;22:589-93. 